\title{
An entropy-based analysis of lane changing behavior: An interactive approach
}

\author{
Caglar Kosun ${ }^{\mathrm{a}}$ and Serhan Ozdemir ${ }^{\mathrm{b}}$ \\ ${ }^{a}$ Department of City and Regional Planning, Izmir Institute of Technology, Izmir, Turkey; ${ }^{b}$ Artificial lytelligence \& Design Laboratory, Department of \\ Mechanical Engineering, Izmir Institute of Technology, Izmir, Turkey.
}

\begin{abstract}
Objectives: As a novelty, this article proposes the nonadditive entropy framework for the description of driver behaviors during lane changing. The authors also state that this entropy framework governs the lane changing behavior in traffic flow in accordance with the long-range vehicular interactions and traffic safety. Methods: The nonadditive entropy framework is the new generalized theory of thermostatistical mechanics. Vehicular interactions during lane changing are considered within this framework. The interactive approach for the lane changing behavior of the drivers is presented in the traffic flow scenarios presented in the article. According to the traffic flow scenarios, 4 categories of traffic flow and driver behaviors are obtained. Through the scenarios, comparative analyses of nonadditive and additive entropy domains are also provided.

Results: Two quadrants of the categories belong to the nonadditive entropy; the rest are involved in the additive entropy domain. Driving behaviors are extracted and the scenarios depict that nonadditivity matches safe driving well, whereas additivity corresponds to unsafe driving. Furthermore, the cooperative traffic system is considered in nonadditivity where the long-range interactions are present. However, the uncooperative traffic system falls into the additivity domain. The analyses also state that there would be possible traffic flow transitions among the quadrants. This article shows that lane changing behavior could be generalized as nonadditive, with additivity as a special case, based on the given traffic conditions.

Conclusions: The nearest and close neighbor models are well within the conventional additive entropy framework. In this article, both the long-range vehicular interactions and safe driving behavior in traffic are handled in the nonadditive entropy domain. It is also inferred that the Tsallis entropy region would correspond to mandatory lane changing behavior, whereas additive and either the extensive or nonextensive entropy region would match discretionary lane changing behavior. This article states that driver behaviors would be in the nonadditive entropy domain to provide a safe traffic stream and hence with vehicle accident prevention in mind.
\end{abstract}

ARTICLE HISTORY

Received 25 April 2016

Accepted 17 June 2016

\section{KEYWORDS}

Nonadditive entropy; traffic flow; driver behavior; lane changing behavior; traffic safety; safe distance

\section{Introduction}

Intervehicle distance in car-following and lane changing behavior could be connected to the safety in the traffic stream. It is expected that the provision of relevant following distance among the vehicles would generate safe driving. In a traffic stream, short- or long-range vehicular interactions can be observed, and these interactions could determine the intervehicle distance. In this stream, those interactions and lane changing behaviors are the 2 important features, and this article examines them within a new modeling perspective; that is, a nonadditive entropy framework.

Lane changing behavior is generally affected by vehicular interactions; however, this is not always observed in real traffic. As Toledo et al. (2007) pointed out, lane changing could be either mandatory or discretionary. For the lane changing behavior models, the lane changing decisions based on interactive driving could reflect a more realistic traffic flow rather than those solely based on independent driving.

Although a number of studies discussed the lane changing behavior models in the literature, interactions among the vehicles during lane changing have not been discussed within the nonadditive entropy context. A real vehicular stream can generally involve long-range interactive driving where properties such as nonergodicity and nonmixing could emerge. Thus, this interactive driving would be well modeled through a nonadditive framework and in this article, for the description of lane changing behavior of drivers, the nonadditive entropy framework is proposed. To the authors' knowledge, this is a first time that the nonadditive entropy framework is intended to qualify the lane changing behavior of drivers in the literature. The nonadditive entropy approach is a novel idea borrowed from nonadditive statistical mechanics and has been used regarding a basic example of vehicular interactions in the study by Kosun and Ozdemir (2014). The authors claim that nonextensive statistical mechanics governs the lane changing behavior in traffic flow in accordance with long-range vehicular interactions. However, once the short-range interactions are in question, BoltzmannGibbs (BG; extensive) thermostatistics would be the underlying statistics to examine the given traffic flow where the unsafe driving would be visible. 
By considering 2-lane traffic flow scenarios in this article, the authors examine the lane changing behaviors in terms of position. The proposed forbidden states, inspired from nonextensive thermostatistics, in the traffic flow scenarios imply safe driving conditions where long-range interactions occur. In this article, both long-range interactions and safety in the traffic stream are handled in the nonadditive entropy framework because the BG statistics would be restrictive. Under a macroscopic viewpoint and certain traffic flow scenarios, driver behaviors are also extracted, and the connection between the behaviors and entropy formalisms is provided. Please note that, though the methodological background section of this article briefly presents the BG and nonextensive (Tsallis) thermostatistics, one may also pursue Tsallis $(1988,2001,2009 a)$, among many others, for the entropy formalisms.

Lane changing models and algorithms have made much progress in the literature and they have considerable applications in the traffic flow field. Lane changing or overtaking models are also still continuing to receive attention. Road capacity and road safety could be potential research interests in those studies. A widely known study by Nagel et al. (1998) proposed 2-lane traffic rules in regard to cellular automata under lane changing behavior. The authors also discussed security and incentive criteria and velocity-based and gap-based rules for lane changing and generated space-time plots and fundamental diagrams. Another study by Knospe et al. (2002) seems representative for the lane changing rules, presenting symmetric and asymmetric models and comparing single-lane and 2-lane traffic. Huang (2002) proposed lane changing rules for a 2-lane highway configuration. In regard to the lane changing rules, incentive and safety criteria are classified and the effects of the 3 parameters in the study are investigated. On lane changing in 2-lane traffic, Nagai et al. (2005) considered traffic states, jamming transitions, and the effect of a bus. The study applies an optimal velocity model and the simulation results are discussed. Research by Qian et al. (2013) established a 3-lane model and focused on the overtaking ratio under different control conditions. On a 4-lane highway, the lane changing and overtaking behaviors of vehicles under mixed traffic conditions are discussed (Chandra and Shukla 2012).

In various studies, the relationships between driver characteristics and overtaking processes are also considered. For example, Mohaymany et al. (2010) found that younger drivers who have less driving experience, and perform more dangerous maneuvers and more risk-taking behaviors are most likely to be at fault in overtaking crashes. The study also recommends that new drivers who are in the first year of driving should be banned from driving on rural roads. Moreover, driver stress and driving performance were investigated in a study by Matthews et al. (1998). A comparison between young male and female drivers during overtaking on a 2-lane highway is made, and the Bayesian methodology is proposed by Vlahogianni and Golias (2012). Statistical analyses were discussed by Bar-Gera and Shinar (2005) to determine whether a tendency of the drivers to pass is correlated with driver attributes. Research by Farah and Toledo (2010) modeled the passing maneuvers in 2 stages; that is, a desire to pass model and a gap acceptance model. For example, the probability of desiring to pass is influenced by the difference between the desired speed of the subject vehicle and the speed of the leading vehicle as well as by the following distance. The individual specific error term that captures the driver characteristics is also related to the probabilities of desiring passing and accepting the available gap. Farah et al. (2009) proposed a passing risk prediction model using regression-based models. They utilized some explanatory variables related to the road geometry, traffic conditions, and driver characteristics.

In a recent review paper by Zheng (2014), lane changing models were comprehensively reviewed in terms of both lane changing decision-making processes and lane changing's impact on surrounding vehicles. Examples of some techniques in lane changing modeling such as cellular automata, Markov processes, and fuzzy logic-based models are presented. The author argues that the existing lane changing decision models consider only one gap or the one nearest the lane changer and the complexity of a lane changing decision-making process needs to be captured by a comprehensive model (Zheng 2014).

It is firmly established that there is strong correlation between driver characteristics and traffic processes such as lane changing and overtaking. Our literature survey serves to prove the lack of studies on not only driver characteristics but the lane changing processes using nonadditive entropy.

Short-range interactions or nearest neighbor models govern the lane changing literature (e.g., Balal et al. 2016; Kesting et al. 2007; Lv et al. 2011; Toledo et al. 2003; Wang et al. 2015). However, though the current literature is explicitly based on short-range interactions, some of the literature deals with interaction potentials that implicitly assume longrange interactions similar to mean field Hamiltonian systems (e.g., Krbálek and Helbing 2004). This naturally leads to the misleading domination of the BG statistical mechanics framework, which strictly assumes the highly idealized conditions of Markovian memory, ergodicity, mixing property, etc. Verification of the invalidity of the BG framework is well hidden in its theoretical assumptions. It would be surprising to find a system with a perfect Markovian memory, a perfect mixing property in phase space, and ergodicity in all of its time series. This assumption is not only purely theoretical but nearly impossible to observe. The BG formulation is an idealized macroscopic view of highly interactive, infinitely many microscopic particles. A corollary of this is the dominion of BG entropy, which is only valid under very special circumstances. The major contribution of the article with respect to traffic research is the substitution of the generalized entropy framework known as Tsallis entropy with the highly improbable concept of BG entropy.

A strong corroboration for this hypothesis comes from research by Kosun and Ozdemir (2016) in which generalized entropy governs the traffic processes. In that work, the authors examined to what extent a given road segment displays BG characteristics. It was found that in none of the generated time series for the observed road segment for a time window of a less than a week yielded an exact BG entropic index parameter of unity. On the contrary, all of the time series generated indices greater than 1, indicating the presence of nonadditivity. This not only proves that it is quite unlikely to get an exact index of $1(q=1)$ but that the traffic interactions led to subadditive Tsallis entropy where long-range interactions reign. In other words, the traffic system for the given road segment exhibited weak chaos, 
non-Markovian memory, and loss of ergodicity as well as the important aspect of the deviation from Gaussianity, as indicated by the $q$-Gaussian distributions.

It is believed that nonadditivity could cover the real traffic stream and specifically lane changing and driver behavior modeling. This framework primarily describes long-range vehicular interactions, including nearest neighborhood interactions as a special case. Hence, both long- and short-range interactive driving could be examined in this generalized framework. Of the vehicular traffic studies, Kosun and Ozdemir (2016) and Koşun (2015) are representative in terms of exhibiting the connection between traffic flow and generalized statistical mechanics. Nonadditivity is discussed in terms of lane changing scenarios and driving safety.

\section{Methodological background}

\section{Boltzmann-Gibbs entropy}

In the BG entropy domain, the total entropy of a system is equal to the sum of the entropies of the $N$ parts of the system and therefore BG entropy has an additive character.

Entropy formulation in the BG approach is as follows (Tsallis 2009a):

$$
\begin{aligned}
& S_{B G}=-k \sum_{i}^{W} p_{i} \ln p_{i} \\
& \sum_{i}^{W} p_{i}=1 \\
& \sum_{i}^{W} p_{i} E_{i}=\langle E\rangle
\end{aligned}
$$

where $E_{i}$ are state energies and $\langle E\rangle$ is the mean energy.

\section{Extensivity and additivity}

Touchette (2002) noted that additivity and extensivity are two different statements. However, they are often confused and utilized to imply one another in the literature. Touchette (2002), on one hand, defined additivity as "a many-body (or joint) physical observable $Q\left(x^{n}\right)$ is said to be additive with respect to two subsystems with states $x^{m}=x_{1} x_{2} \ldots x_{m}$ and $x^{n-m}=$ $x_{m+1} x_{m+2} \ldots x_{n}$ if $Q\left(x^{n}\right)=Q\left(x^{m}\right)+Q\left(x^{n-m}\right)$ " (p. 85), on the other hand, defines extensivity as "a joint observable $Q\left(x^{n}\right)$ is extensive if the $Q$-density, defined by the ratio $Q\left(x^{n}\right) / n$, reaches a constant in the limit $n \rightarrow \infty$ ” (p. 87).

An entropy $S(N)$ of a given system constituted by $N$ vehicles (elements) is extensive when Eq. (4) is satisfied. Otherwise, the system entropy is nonextensive.

$$
0<\lim _{N \rightarrow \infty} \frac{S(N)}{N}<\infty
$$

where $S$ is entropy and $N$ is the number of vehicles.

\section{Tsallis entropy}

This section introduces the Tsallis entropy; that is, generalization of BG entropy, known as nonadditive entropy. In 1988, Tsallis postulated the nonadditive entropy formalism for the generalization of BG thermostatistics. Boltzmann-Gibbs entropy might be a convenient expression for particular cases; however, it has some limitations with regard to, for example, systems involving ergodicity breaking and long-range interactions. Such systems may not be expounded by BG entropy.

In the new formalism, Tsallis introduced the $q$ entropic index into the entropy formulation, which is written in the following form (Tsallis 2009a):

$$
S_{q}=k \frac{1-\sum_{i=1}^{W} p_{i}^{q}}{q-1} \quad\left(\sum_{i}^{W} p_{i}=1 ; k>0\right)
$$

where $k$ is the Boltzmann constant, $q$ is the entropic index, and $p_{i}$ is the state probability.

For the case $q=1$, Tsallis entropy represents BG entropy $\left(S_{1}=S_{q}\right)$. For the discrete case, it is obtained as follows (Tsallis 2009a):

$$
\lim _{q \rightarrow 1} S_{q}=S_{1}=-k \sum_{i=1}^{W} p_{i} \ln p_{i}
$$

If all of the probabilities are equal, the entropy $S_{q}$ is obtained as

$$
S_{q}=k \ln _{q} W \quad\left(S_{1}=S_{B G}\right)
$$

\section{Nonadditivity and $q$ entropic index}

Suppose that A and B are two probabilistically independent subsystems and if the joint probability satisfies $p_{i j}^{A+B}=$ $p_{i}^{A} p_{j}^{B}(\forall(i j))$, and verified that, for example in Tsallis (2009b).

$$
\frac{S_{q}(A+B)}{k}=\frac{S_{q}(A)}{k}+\frac{S_{q}(B)}{k}+(1-q) \frac{S_{q}(A)}{k} \frac{S_{q}(B)}{k}
$$

This is also rearranged as

$$
S_{q}(A+B)=S_{q}(A)+S_{q}(B)+\frac{(1-q)}{k} S_{q}(A) S_{q}(B)
$$

For $q=1$, the additivity of BG entropy is obtained for any finite value of $k$.

$$
S_{B G}(A+B)=S_{B G}(A)+S_{B G}(B)
$$

In the case of $q \neq 1$ the entropy $S_{q}$ is nonadditive. This case is classified according to $q$ value (Tsallis 2009a).

If $q<1, S_{q}(A+B) \geq S_{q}(A)+S_{q}(B)$ and the system is called superadditive.

If $q>1, S_{q}(A+B) \leq S_{q}(A)+S_{q}(B)$ and the system is called subadditive.

BG entropy is additive, whereas Tsallis entropy (generalization of BG entropy) is nonadditive (Tsallis 2009a). The additive (BG) entropy could be either extensive or nonextensive, and the same idea is valid for nonadditive entropy. Thus, nonadditive entropy (Tsallis thermostatistics) could be either extensive or nonextensive. This is shown in Figures 1-3. Nonadditive entropy is traditionally called nonextensive entropy (Tsallis 
ADDITIVE SUBADDITIVE

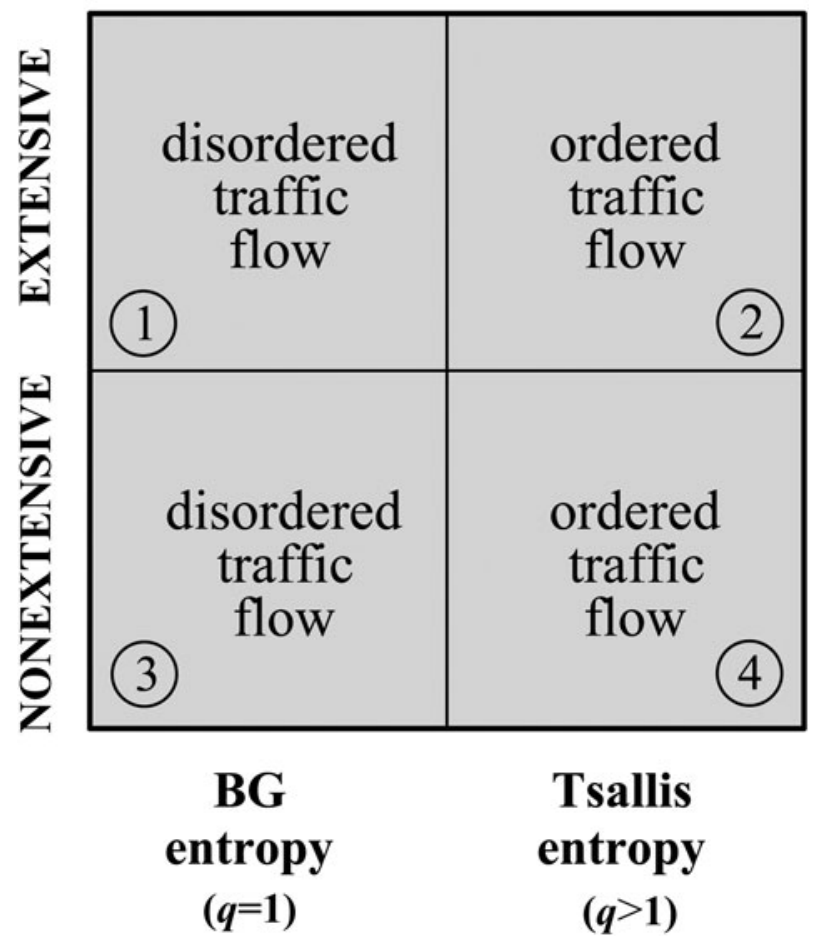

Figure 1. Classification of traffic flow in terms of entropic additivity and entropic extensivity.

2009a). However, it is clearly represented in generalized thermostatistical literature that they are conceptually separate identities.

The concepts of superadditivity and subadditivity are subsumed under nonadditivity. As explained in Tsallis (2001), q $>1$ privileges frequent events, whereas $q<1$ privileges rare events. Both super- and subadditivity involve short- and longrange interactions. A traffic flow that is subadditive in nature is a result of the interactions where safety is the major concern so that lane violations are avoided. On the other hand, a compulsory and/or urgent lane violation such as by an ambulance would give rise to superadditive traffic flow. In this work, the subadditive nature of the traffic flow is considered, where traffic safety is the main concern.

\section{Scenario setup}

In this article, we conduct a scenario-based study and assume that there is a 2-lane highway section. The lane changing behaviors of the vehicles are investigated under the nonadditive entropy and additive entropy frameworks only for the $q$ value of unity. Four scenarios are discussed and illustrated for common vehicles such as motorbikes and cars.

Additive behavior in traffic does not take into consideration whether the lanes are occupied or not, where the drivers travel ignoring safety and traffic regulations. On the contrary, driver behavior that falls into a subadditive property of a greater nonadditivity domain would consider long-range interactions and carefully choose the lanes. Nonextensivity could be related to the loss of allowable states. If the allowable states are no longer proportional to the number of vehicles, nonextensivity emerges
ADDITIVE SUBADDITIVE

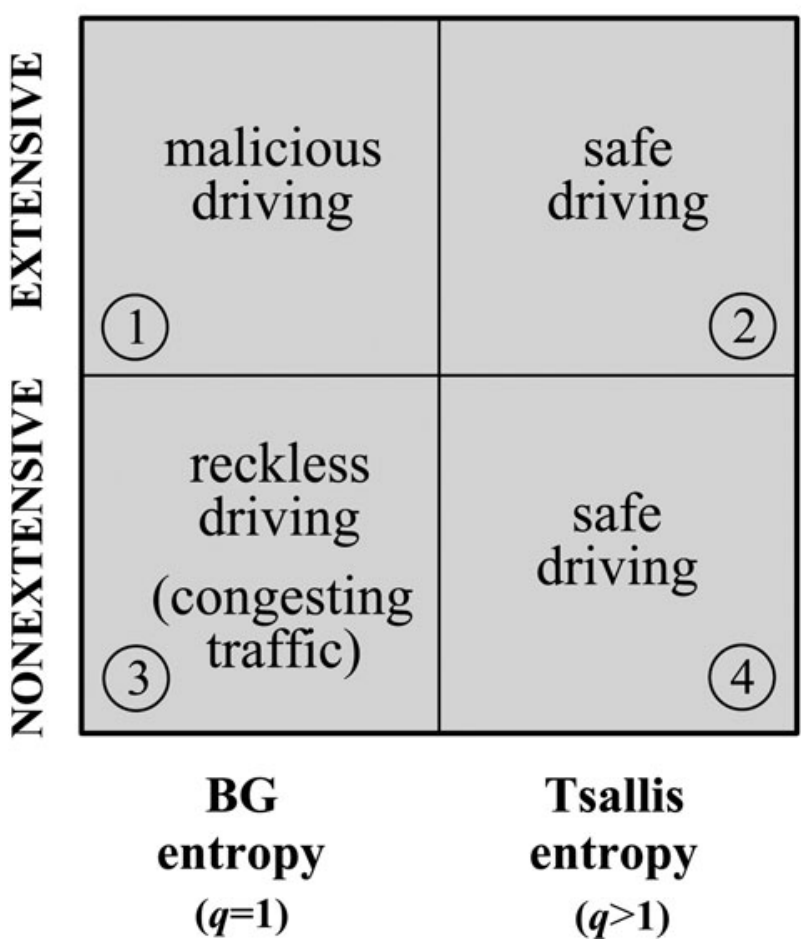

Figure 2. Representation of driver behaviors in terms of entropic additivity and entropic extensivity.

and the correlations tend to be stronger. However, in extensive traffic conditions, entropic extensivity exists as long as this proportionality remains finite. Extensivity property is replaced by nonextensivity with the emergence of a certain saturation. Some introductory theoretical arguments are given in Tsallis (2001). As stated in Tsallis (2001), the thermodynamics of shortrange interactions is extensive where the BG statistics $(q=1)$ exists. Strongly disordered or chaotic systems are encountered in the BG domain where they have the lowest predictability. In contrast, the thermodynamics of long-range interactive systems is nonextensive (generalized thermostatistical formalism) and those systems are more predictable (intermediate predictability), with multifractal and non-Markovian properties (Tsallis 2001).

\section{Traffic flow analysis in terms of entropy}

In this section, the extensivity/nonextensivity and additivity/nonadditivity concepts are classified into 4 categories within the notion of ordered and disordered traffic flows. This classification is summarized in Figure 1. These categories are called quadrants and are labeled from 1 through 4 in connection with the scenarios in Figure 4. The descriptions of the quadrants are given below.

Quadrant 1: Consider an extensive and additive traffic flow in BG domain (Figure 1). During lane changing behavior, all drivers can choose all states without taking care of any longrange interactions. This driver behavior can be observed in disordered traffic flow.

Quadrant 2: In the subadditive and extensive quadrant (Figure 1), the characteristics of prudent drivers may 


\section{ADDITIVE SUBADDITIVE}

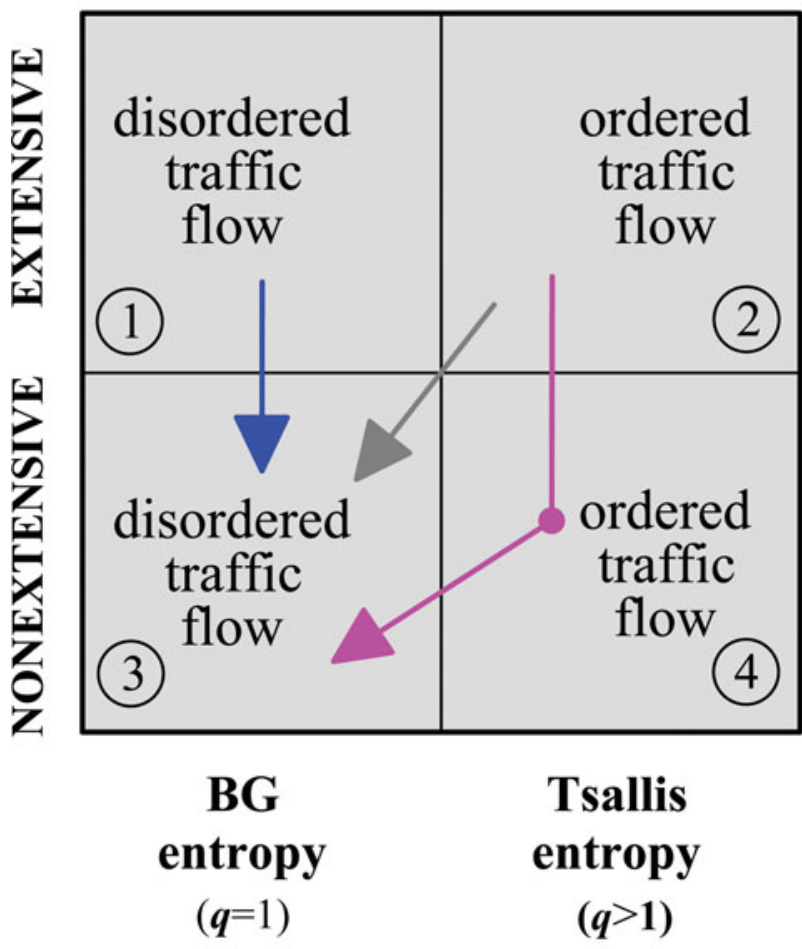

traffic transitions

\section{from 1 to 3}

from 2 to 3

$\longrightarrow$ from 2 to 4 , and then 3

Figure 3. Transitions among traffic flow classifications in terms of entropic additivity and entropic extensivity.

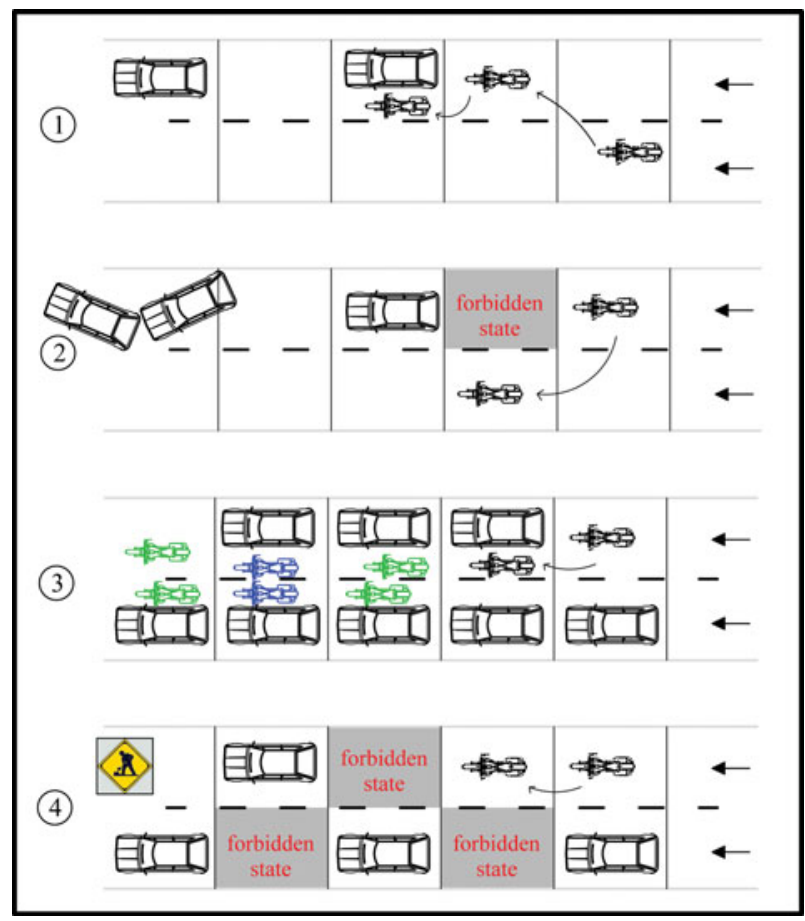

Figure 4. Driver behaviors in the given traffic flow scenarios. give rise to entropic nonadditivity. These drivers take care not to bother other drivers during lane changes. Though the number of vehicles tends to infinity, entropic extensivity exists because allowable states are proportional to $N$, as in Eq. (4), until a certain saturation. In this quadrant, traffic flow is said to be ordered.

Quadrant 3: In the additive and nonextensive quadrant, entropic additivity would exist due to reckless drivers, in the sense that individual entropies of the drivers are additive due to lack of consideration of others (very short-range interactions) during lane changing. Nonextensivity emerges because allowable states are no longer proportional to $N$. The traffic flow in this quadrant is thought to be disordered because a certain saturation is reached due to the loss of extensivity. At worst, traffic jams are expected to occur.

Quadrant 4: When a congested flow is imminent, the system entropy is nonextensive due to loss of allowable states, whereas the entropy is still considered to be subadditive because drivers are even more prudent when changing lanes. The traffic flow would be inevitably ordered in the system. Quadrant 4 may also lead to initiation of a traffic jam, and drivers will gradually consider forbidden states allowable, but still observing the usual codes of conduct in traffic flow.

As a result, the traffic stream is divided in 2 groups-that is, ordered and disordered-corresponding to Tsallis and BG entropy formalisms, respectively.

\section{Traffic flow scenarios and driver behavior}

Through the aforementioned quadrant descriptions, lane changing scenarios can be illustrated as in Figure 4. One can describe driver behavior under these classifications. For example, the behavior of a motorbike driver in traffic is displayed in Figure 4 in terms of entropy.

In Figure 4, 4 different driver behaviors are extracted based on the traffic flow classifications. For all 4 scenarios depicted in Figure 4, suppose that the leading vehicle has a constant velocity and travels in the right lane. However, the motorbike, the following vehicle, tries to overtake the car. The driver behavior designation in Figure 2 is formed after analyzing each scenario in Figure 4.

In driver behavior 1 (Figure 4), the following vehicle (motorbike) intends to overtake the car with constant velocity. When overtaking, the motorbike does not change lanes and prefers to travel in the same state with the car rather than traveling in the adjoining state of the overtaking route. We call this driving behavior malicious driving. The driver can choose any possible state with equal probability and the system is defined in the additive and extensive quadrant, including short-range interactions.

In driver behavior 2, long-range interactions are present. The motorbike driver follows the leading vehicle until the driver observes a roadblock ahead. After observing it, the motorbike passes the adjoining state of its own and follows a new route. This driver behavior could be classified in the subadditive and extensive quadrant (Figure 2). Here, safe driving behavior emerges due to the long-range interactions. 
In driver behavior 3 , the motorbike again approaches the leading car to overtake. Similar to the first driving scenario, the motorbike driver chooses the state of the leading vehicle. In this scenario, the entropy is in the nonextensive and additive quadrant, and reckless driving behavior appears. The traffic flow is congested and the allowable states are substantially occupied.

In driver behavior 4, the motorbike driver is more prudent in overtaking the car. That is, the driver does not want to choose the state behind the car and is reluctant to pass into the state of the leading vehicle. Furthermore, the motorbike cannot overtake the car due to the roadblock ahead; therefore, the entropy is subadditive. Moreover, the other states cannot compensate for the increase in $N$ (vehicles) and entropic extensivity is violated. In the fourth behavior, the motorbike driver shows a safe driving profile. As in driver behavior 2, the interactions are described as long-range. Therefore, it is safe to say that when the long-range interactions are in question, the nonadditive entropy region may emerge because the states choices are not proportional to $1 / \mathrm{W}$ (Eq. (7)).

As a result, scenario 1 and scenario 3 are classified as disordered because the motorbike driver can choose any state ignoring traffic safety. Thus, the total entropy of each driver in these scenarios is additive. The main difference between scenario 1 and scenario 3 is the traffic density. The traffic density is related to Eq. (4). By increasing the number of vehicles, the proportion of the entropy and the number of vehicles may not be finite and this situation results in nonextensivity. Scenario 2 and scenario 4 could be discussed in the other thermostatistical perspective (generalized thermostatistics). In these scenarios, longrange interactions occur, and the system would be in the ordered category. The drivers now move by considering safety conditions and the other drivers in traffic.

In the light of lane changing behaviors, a comparative analysis of the BG and Tsallis entropy approaches for the given traffic flow scenarios is carried out in this study. The authors assert that the autonomous and short-range behavior of vehicles fall into BG entropy $(q=1)$, whereas the long-range interactions of the vehicles would fall into Tsallis entropy $(q \neq 1)$. Moreover, malicious and reckless driving behaviors, which are subsumed under unsafe driving, fall into the BG domain, whereas safe driving reflects the Tsallis domain.

Safe driving and the $q$ entropic index are highly interconnected because the $q$ index is an indicator of driving characteristics that represent interactions in traffic. Any transition in traffic flow from the BG domain to the Tsallis entropy (subadditive) domain indicates a deviation in the $q$ value from unity. This deviation in the $q$ value depends on, for example, long-range interactions, observing safe distance, safety rules, etc. However, when the traffic flow is in the BG (additivity) domain, reckless and malicious driving behaviors emerge, leading to unsafe driving, making the traffic disordered and uncooperative.

Though lane changing dynamics were explained with regard to a motorbike and a car, this analogy may be extended to vehicle-to-vehicle interactions. For example, in the motorbike analogy, both the car and the motorbike may fit in a single lane, which is a clear indication of the violation of the nonadditivity property. The property of additivity could be represented in a multitude of ways in vehicle movements. Hence, the idea of the additivity property in traffic flow is by no means constricted to a motorbike-car pair but could be extended to vehicle-to-vehicle interactions. Any lane violation scenario involving reckless (or malicious) driving behavior would yield additivity. For example, zigzagging on a highway, disregarding safety and traffic regulations at all times, results in additivity where the occupation of a single lane by 2 vehicles ought not necessarily be observed. Even a partial violation of a lane by a vehicle that is already occupied by another vehicle would suffice to induce additivity.

\section{Discussion}

Figure 2 can be interpreted by observing Figures 1 and 4 and presents the driver behaviors in terms of entropy. According to Figure 2, entropy of the system constituted by $N$ elements is extensive in the first row and is between $0<S(N) / N<\infty$ $\lim _{N \rightarrow \infty}$, and the entropy is additive in the first column where $q=1$. In each entropy domain (BG or Tsallis), loss of allowable states results in different driving behavior. As a result, in light of the Figures 1-4, it can be pointed out that the traffic system is both cooperative and uncooperative. In the cooperative traffic system, vehicles are traveling in harmony with each other. Some of the states are forbidden automatically, and random movements are decreasing. Cooperation moves the traffic system into the Tsallis entropy domain and traffic is considered within long-range relationships. In contrast, a traffic system that is uncooperative can display random movements provided that no collisions among the vehicles are allowed. The uncooperative traffic system is considered in BG statistical mechanics where short-range interactions are present.

The analyses also show that there would be transitions among the quadrants of the traffic flow, which are explained below. The possible transitions are shown in Figure 3.

- Transition from 2 to 3: From the viewpoint of the transition from 2 to 3, prudent drivers in quadrant 2 do not bother other drivers, especially when long-range interactions are present. This driver behavior is placed in the extensive and subadditive quadrant. When the traffic flow gets congested, capacity is slowly reached and state choices begin to be made. Concurrently, nonextensivity emerges and the drivers could also attempt to occupy empty spaces. The system remains in the nonextensive and additive quadrant and the entropy decreases as well.

- Transition from 2 to 4 and then 3: If traffic congestion is beginning to appear where prudent drivers travel within the subadditive and extensive quadrant, the traffic can tend toward the nonextensive and subadditive zone. Due to increasing congestion, the drivers change their own behaviors and want to occupy the adjacent states ignoring all interactions. Therefore, the traffic flow is said to be additive and nonextensive until traffic is literally congested; that is, nonextensive and additive.

- Transition from 1 to 3: Suppose that the traffic flow is in the additive and extensive quadrant, strictly in the BG domain. If the traffic is congested and the drivers are positioned bumper to bumper at worst, the traffic flow system is transferred to the additive and nonextensive quadrant.

As also inferred from the transition scenarios above, the nonadditive entropy framework is the new generalized theory of thermostatistical mechanics. It is not meant to replace the 
celebrated BG statistical mechanics but instead to generalize it. Because BG statistical mechanics is heavily dependent on a short-range interactions assumption, this concept may not find a niche in real-life applications or modeling. Incidentally, many traffic models involve either short-range interactions or no interactions at all.

This article has addressed the lane changing behavior of drivers. Lane changing models and algorithms are widely discussed in the literature. These models have implemented various techniques. A nonadditive entropy framework is new to traffic modeling arena. This framework was first used in vehicular interactions in Kosun and Ozdemir (2014). This was the first application of the nonextensive framework in traffic flow. Similarly, the authors of the present article used the same framework to discuss the lane changing behaviors of drivers considering correlations.

The basis of this work is that the lane changing process involves interactions. These interactions may involve nearest neighbor interactions but those neighbors may be at least a mile away. The nearest and close neighbor models are well within the BG statistics, deliberately or not. However, the authors claim that nonextensive statistical mechanics governs the lane changing behavior in traffic flow in accordance with the long-range vehicular interactions in this article.

The underlying principles are complex and stem from statistical mechanics. The readers may find Tsallis (2009a, 2009b) interesting reading penned by the founder of the theory. Based on this theory, a series of scenarios was provided to explain the 4 possible cases. Then a comparative analysis of the additive and nonadditive entropy approaches for the given traffic flow scenarios was provided. The authors hence suggest that the autonomous (reckless) and short-range behavior of the vehicles falls into the additive entropy domain, whereas the long-range interactions (respectful of approaching traffic) of the vehicles would fall into the nonadditive entropy domain.

At macroscopic level, the primary effort of this study is to discuss the nonextensive thermostatistics framework to describe lane changing and driver behavior. Depending on the given scenarios, the Tsallis entropy region would correspond to mandatory lane changing behavior, whereas the BG entropy region would match discretionary lane changing behavior. Hence, Tsallis entropy (subadditive) describes the safe distance and safe driving behavior in the traffic flow. A consequence is that the $q$ entropic index could represent the safety level in the traffic stream.

\section{Acknowledgments}

This article is derived from part of a PhD dissertation, entitled The Use of Nonextensive Framework in Connection with Traffic Flow, carried out at the Izmir Institute of Technology Graduate School of Engineering and Sciences (2015).

\section{References}

Balal E, Cheu RL, Sarkodie-Gyan T. A binary decision model for discretionary lane changing move based on fuzzy inference system. Transp Res Part C Emerg Technol. 2016;67:47-61.
Bar-Gera H, Shinar D. The tendency of drivers to pass other vehicles. Transp Res Part F Traffic Psychol Behav. 2005;8:429-439.

Chandra S, Shukla S. Overtaking behavior on divided highways under mixed traffic conditions. Proc Soc Behav Sci. 2012;43:313-322.

Farah H, Bekhor S, Polus A. Risk evaluation by modeling of passing behavior on two-lane rural highways. Accid Anal Prev. 2009;41:887-894.

Farah H, Toledo T. Passing behavior on two-lane highways. Transp Res Part F Traffic Psychol Behav. 2010;13:355-364.

Huang DW. Lane-changing behavior on highways. Phys Rev E. 2002;66:026124-1.026124-5.

Kesting A, Treiber M, Helbing D. General lane-changing model MOBIL for car-following models. Transp Res Rec. 2007;1999:86-94.

Knospe W, Santen L, Schadschneider A, Schreckenberg M. A realistic two-lane traffic model for highway traffic. J Phys A-Math Gen. 2002;35:3369-3388.

Koşun Ç. The Use of Nonextensive Framework in Connection with Traffic Flow [PhD dissertation]. Izmir, Turkey: Department of City and Regional Planning, Izmir Institute of Technology Graduate School of Engineering and Sciences; 2015.

Kosun C, Ozdemir S. Entropy in terms of vehicular distance under driving constraints. Paper presented at: International Conference on Mathematics and Computers in Science and Industry; September 13-15, 2014; Varna, Bulgaria.

Kosun C, Ozdemir S. A superstatistical model of vehicular traffic flow. Physica A. 2016;444:466-475.

Krbálek M, Helbing D. Determination of interaction potentials in freeway traffic from steady-state statistics. Physica A. 2004;333:370-378.

Lv W, Song WG, Fang ZM. Three-lane changing behaviour simulation using a modified optimal velocity model. Physica A. 2011;390:23032314.

Matthews G, Dorn L, Hoyes TW, Davies DR, Glendon AI, Taylor RG. Driver stress and performance on a driving simulator. J Hum Factor Ergon Soc. 1998;40:136-149.

Mohaymany AS, Kashani AT, Ranjbari A. Identifying driver characteristics influencing overtaking crashes. Traffic Inj Prev. 2010;11:411416.

Nagai R, Nagatani T, Taniguchi N. Traffic states and jamming transitions induced by a bus in two-lane traffic flow. Physica A. 2005;350:548562.

Nagel K, Wolf DE, Wagner P, Simon P. Two-lane traffic rules for cellular automata: a systematic approach. Phys Rev E. 1998;58:1425-1437.

Qian Y, Luo J, Zeng J, Shao X, Guo W. Study on security features of freeway traffic flow with cellular automata model-taking the number of overtake as an example. Measurement. 2013;46:20352042.

Toledo T, Koutsopoulos H, Ben-Akiva M. Modeling integrated lanechanging behavior. Transp Res Rec. 2003;1857:30-38.

Toledo T, Koutsopoulos HN, Ben-Akiva M. Integrated driving behavior modeling. Transp Res Part C Emerg Technol. 2007;15(2):96-112.

Touchette $\mathrm{H}$. When is a quantity additive, and when is it extensive? Physica A. 2002;305:84-88.

Tsallis C. Possible generalization of Boltzmann-Gibbs statistics. J Stat Phys. 1988;52:479-487.

Tsallis C. Nonextensive statistical mechanics and thermodynamics: historical background and present status. In: Sumiyoshi A, Okamoto Y, eds. Nonextensive Statistical Mechanics and Its Applications. New York, NY: Springer; 2001:3-98.

Tsallis C. Introduction to Nonextensive Statistical Mechanics: Approaching a Complex World. New York, NY: Springer; 2009a.

Tsallis C. Nonadditive entropy and nonextensive statistical mechanics-an overview after 20 years. Braz J Phys. 2009b;39:337-356.

Vlahogianni EI, Golias JC. Bayesian modeling of the microscopic traffic characteristics of overtaking in two-lane highways. Transp Res Part F Traffic Psychol Behav. 2012;15:348-357.

Wang M, Hoogendoorn SP, Daamen W, van Arem B, Happee R. Game theoretic approach for predictive lane-changing and car-following control. Transp Res Part C Emerg Technol. 2015;58:73-92.

Zheng Z. Recent developments and research needs in modeling lane changing. Transp Res Part B Meth. 2014;60:16-32. 